\title{
Gallbladder perforation with formation of hepatic subcapsular biloma, treated with endoscopic nasobiliary drainage
}

A 66-year-old man presented with right upper quadrant pain and progressive dyspnea for 2 days. He had history of chronic obstructive pulmonary disease and chronic kidney disease. On admission, he was febrile and had sinus tachycardia. Laboratory findings showed an elevated white blood cell count of $18.01 \times 10^{9} / \mathrm{L}$ with $92.1 \%$ neutrophils. Liver function tests revealed normal alanine aminotransferase (14U/L [3-37U/L]), normal total bilirubin $(0.6 \mathrm{mg} / \mathrm{dL}[0-1.0 \mathrm{mg} / \mathrm{dL}])$, and elevated alkaline phosphatase (157 U/L [39-120 U/L]). Ultrasonography showed a big encapsulated cystic lesion in right upper quadrant of abdomen, with amorphous echogenic material within the lesion. The gallbladder was small in size and a 0.9 -cm gallstone was noted ( $\bullet$ Fig. 1 ).

An abdominal computed tomography (CT) scan revealed a hepatic subcapsular cystic lesion, which was communicating with the gallbladder ( $\bullet$ Fig. 2).

Infection was initially controlled by percutaneous drainage of the lesion and administration of antibiotics. However, due to concern about the high surgical risk of the patient and the persistent large amounts (500-600 mL/day) of bile recovered from the percutaneous drainage, an endoscopic retrograde cholangiography (ERC) with endoscopic nasobiliary drainage (ENBD) was carried out, which showed extravasation of contrast medium from the gallbladder into the perihepatic area ( $\bullet$ Fig. 3).

After this procedure, the amount of bile in the percutaneous drainage decreased gradually. Two weeks later, ERC via ENBD revealed cessation of leakage ( Fig. 4 ).

The patient was discharged uneventfully after removal of the catheters.

Gallbladder perforation is a rare but lifethreatening condition and usually caused by acute cholecystitis or trauma. Niemeier classified gallbladder perforation as: free perforation to the peritoneal cavity (type I); pericholecystic abscess or localized perforation (type II); and cholecystoenteric fistula (type III) [1]. The therapeutic modalities include percutaneous gallbladder drainage and open cholecystectomy. A previous report has even demonstrated successful treatment of post-traumatic

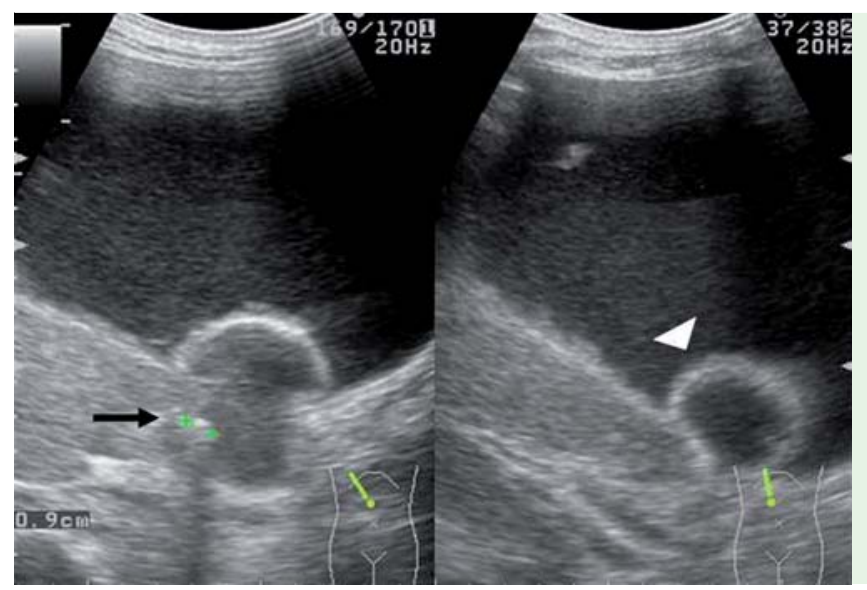

Fig. 1 Ultrasonography showing a big encapsulated cystic lesion located in right upper quadrant. Note the small gallbladder (arrowhead) and the 0.9-cm stone (arrow).

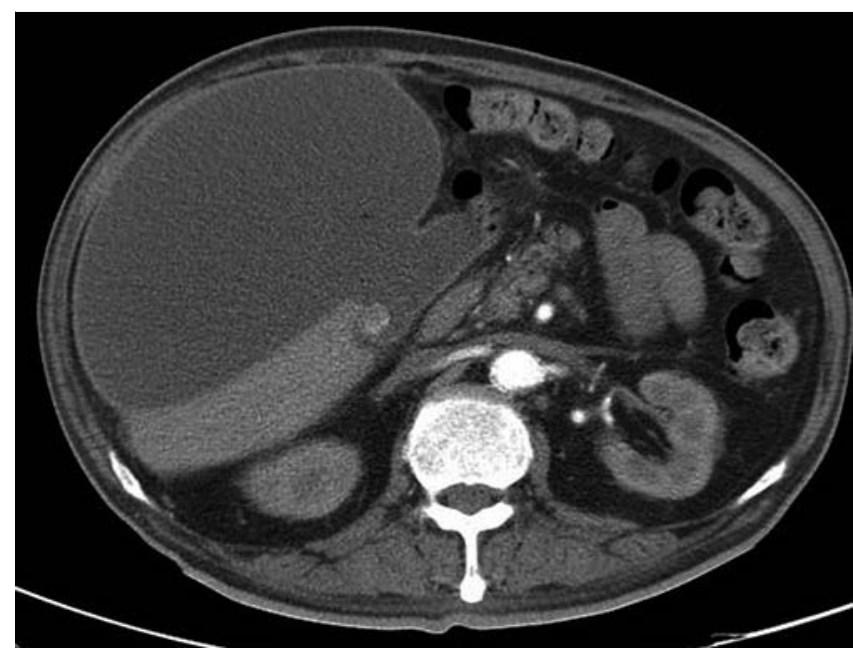

Fig. 2 Computed tomography (CT) scan demonstrating gallbladder perforation with hepatic subcapsular biloma formation.

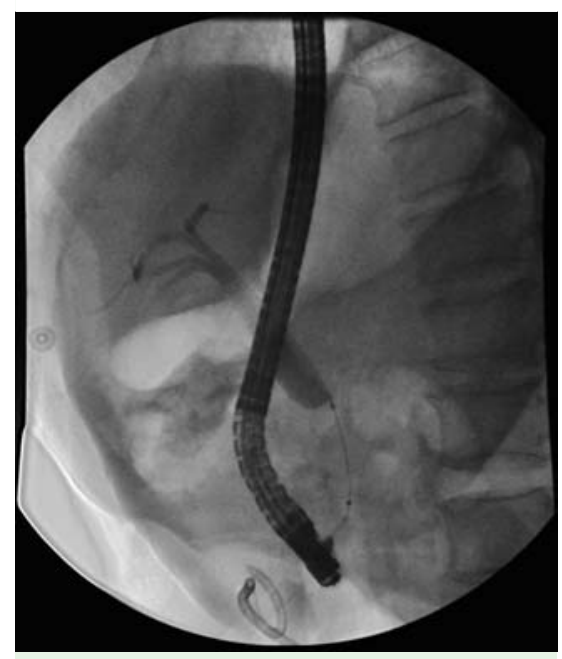

Fig. 3 Endoscopic retrograde cholangiography (ERC) showing extravasation of contrast medium from the gallbladder into the perihepatic area.

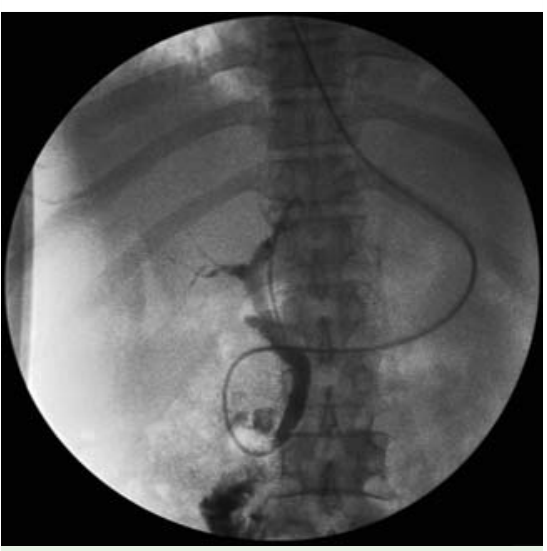

Fig. 4 Cholangiography two weeks later (via endoscopic nasobiliary drainage) did not show any extravasation of contrast medium. 
gallbladder perforation by endoscopic biliary stent placement [2]. Our case presented a rare complication of gallbladder perforation, which was treated successfully with EBND.

Competing interests: None

Endoscopy_UCTN_Code_TTT_1AQ_2AJ
M. C. Tsai, T. H. Chen, M. H. Chang,

T. Y. Chen, C. C. Lin

Department of Internal Medicine, Chung Shan Medical University Hospital and Institute of Medicine of Chung Shan Medical University, Taichung, Taiwan

\section{References}

1 Niemeier OW. Acute free perforation of the gallbladder. Ann Surg 1934; 99: 922 - 924

2 Sugiyama M, Abe N, Masaki T et al. Endoscopic biliary stent placement for treatment of gallbladder perforation due to blunt abdominal injury. Gastrointest Endosc 2000; 52: $275-277$
Bibliography

DOI $10.1055 / \mathrm{s}-0030-1255709$

Endoscopy 2010; 42: E206-E207

(c) Georg Thieme Verlag KG Stuttgart · New York . ISSN 0013-726X

Corresponding author

\section{C. Lin}

Department of Internal Medicine

Chung Shan Medical University Hospital

No. 110 Chien-Kuo N. Rd Sec. 1

Taichung 402

Taiwan

Fax: +886-4-24739220

forest65@ms8.hinet.net 